\title{
Una nueva especie de pez del género Bryconamericus (Ostariophysi: Characidae) del río Magdalena, con una clave para las especies de Colombia
}

\author{
César Román-Valencia, James A. Vanegas-Ríos \& Raquel I. Ruiz-C. \\ Universidad del Quindío, Laboratorio de Ictiología, A. A. 2639, Armenia, Colombia. ceroman@uniquindio.edu.co, \\ anyelovr@gmail.com, raquelivveth.ruizcalderon@gmail.com
}

Recibido 28-I-2008. Corregido 30-VI-2008. Aceptado 31-VII-2008.

\begin{abstract}
A new species of fish of the genus Bryconamericus (Ostariophysi: Characidae) from the Magdalena river, with a key to Colombian species. Based on a series of 90 specimens, and using morphometric, meristic and osteological characters, a new species of the genus Bryconamericus is described from Pontesuela Creek at the mouth of Coclina Creek in the middle Magdalena river Basin of Colombia. We use box graphs with $99 \%$ confidence intervals to illustrate the extent of meristic character overlap and to help distinguish this new taxon from other species of the Magdalena River. The species can be distinguished from all congeners by the combination of the following characters: the higher number of lateral line scales (50-54 vs. 30-50, except B. terrabensis with 47-54); more scales between the lateral line and the dorsal fin origin (9-11 vs. 4-9); more scales between the lateral line and the pelvic fin origin (8-10 vs. 2-8); more scales between lateral line and anal fin origin (8-10 vs. 2-8, except B. terrabensis with 6-9) and more predorsal scales (16-18 vs. 9-16, except B. huilae with 14-19 scales, B. galvisi with 12-17 and B. tolimae with 13-17). Rev. Biol. Trop. 56 (4): 1749-1763. Epub 2008 December 12.
\end{abstract}

Key Words: Bryconamericus sp. nov., Characid fish, Magdalena, South America.

En las revisiones y trabajos relacionados con Bryconamericus Eigenmann (Almiron et al. 2004, Eigenmann 1927, Ferreira y Carvajal 2007, Ferreira y Lima 2006, Vari y Siebert 1990, Bizerril y Auraujo 1992, Casciotta et al. 2004, Langeani et al. 2005, Lima et al. 2003, Lima et al. 2004, Malabarba y Kindel 1995, Miquelarena y Aquino 1995, RománValencia 2002, 2003a-c, 2004, 2005, Silva 2004, Román-Valencia et al. 2008) se reconocen 78 especies (número que puede aumentar) e incluye a Knodus Eigenmann, con amplia distribución desde Costa Rica en Centro América hasta Argentina en Suramérica. También, existe un análisis filogenético de las especies de Bryconamericus para Centro América con ADNmt, donde se plantea su monofilia (C. Román-Valencia \& J.A. Vanegas-Ríos, en prep). Vanegas-Ríos (2008) demuestra que
Bryconamericus distribuidas en Centro América y noroccidente de Suramérica forman un grupo monofilético, soportado por tres sinapomorfías asociadas con el aparato de Weber, ceratohial anterior y cartílago posterior del metapterigoides. Román-Valencia (2003a, 2004) reconoce 22 especies válidas de Bryconamericus para Colombia: B. alpha Eigenmann, Henn \& Wilson, B. andresoi Román-Valencia, B. carlosi Román-Valencia, B. caucanus Eigenmann, B. cismontanus Eigenmann, Henn \& Wilson, B. cristiani Román-Valencia, B. dahli RománValencia, B. emperador Eigenmann \& Ogler, B. galvisi Román-Valencia, B. guaytarae Eigenmann, Henn \& Wilson, B. guizae RománValencia, $B$. heteresthes Eigenmann, $B$. huilae Román-Valencia, B. hypopterus Fowler, $B$. ichoensis Román-Valencia, B. loisae Géry, B. miraensis Fowler, B. multiradiatus Dahl, 
B. orteguasae Fowler, B. plutarcoi RománValencia, $B$. peruanus Müller \& Troschel y $B$. tolimae Eigenmann.

El propósito de éste artículo es describir una nueva especie de Bryconamericus del sistema del río Magdalena y es parte de la revisión que continúa el autor (C.R-V.) sobre las especies de Bryconamericus para el norte de Sudamérica.

\section{MATERIAL Y MÉTODOS}

Las recolecciones de los peces en la localidad típica del nuevo taxon, Quebrada La Pontesuela en la boca de la quebrada Coclina en el Magdalena medio, se realizaron con un método consistente en arrastres con una malla fina (ojo de malla 6.0 por $3.5 \mathrm{~mm}$ ) de $3.85 \mathrm{~m}$ de largo por $1.65 \mathrm{~m}$ de ancho. Los arrastres se hicieron en un sólo biotopo: zona litoral de remanso, a favor de la corriente, con una frecuencia de dos a tres arrastres por sitio. Se registró la coloración en vivo y se fijaron in situ con formol al 10\%. Se preservó posteriormente en alcohol al 70\%, se depositó en el laboratorio de Ictiología del Departamento de Biología, Universidad del Quindío, Armenia, Colombia (IUQ). En total se analizaron 90 ejemplares correspondientes al nuevo taxon. Se examinó material de comparación depositado en: museo de Biología, Instituto de Zoología Tropical de la Universidad Central de Venezuela, Caracas (MBUCV), Field Museum of Natural History de Chicago (FMNH), Instituto de Investigaciones Biológicas "Alexander Von Humboldt", Villa de Leyva, Boyacá (IaVH). En la localidad de muestreo, se hicieron determinaciones diurnas de las siguientes variables: oxígeno disuelto, temperatura superficial del agua y del ambiente determinado con oxímetro, $\mathrm{pH}$ con potenciómetro. Tipo de sustrato y color del ambiente acuático, calificado por observación directa, ancho y profundidad con flexómetro y vareta graduada en $\mathrm{cm}$. Coordenadas y altitud con un sistema electrónico portátil de posición global (GPS) 4000XL.

Las medidas de los ejemplares se tomaron con un calibrador digital, hasta centésimas de mm. Medidas y conteos se realizaron sobre el lado izquierdo de los ejemplares (Cuadro 1), excepto cuando éstos estaban deteriorados en tal lado. Se sigue a Fink y Weitzman (1974) para la obtención de medidas con las adiciones de Román-Valencia (2003a). Y se amplió con datos comparativos merísticos de las especies de Bryconamericus descritas hasta el momento (cuadro 2) con base en las descripciones originales. El número de ejemplares en material examinado se da entre paréntesis. Se utilizó un gráfico de cajas y de intervalos de confianza al 99\% para ilustrar la medida merística que mostrara menor solapamiento y distinguiera parcialmente al nuevo taxon de las otras especies del río Magdalena. Las pruebas aquí aplicadas fueron llevadas a cabo para ejemplares adultos, en el programa SPSS 9.0 bajo Windows.

Las observaciones de las estructuras óseas y cartílagos se hicieron sobre ejemplares clareados y teñidos (C. y T.) de acuerdo a modificaciones del método descrito por Taylor y Van Dyke (1985). La nomenclatura de huesos se basó en Weitzman (1962), Murray (2004) y Ruiz-C y Román-Valencia (2006).

\section{Material de comparación examinado}

Bryconamericus caucanus. (Véase FMNH 56230 paratipos y IUQ 335, 336, 337, 340, 341, 342, 344, 346, 351, 352, 378, 379 en Román-Valencia, 2003a). MBUCV 27914, (15), Cesar, río Hurtado, afluente del río Cesar, cuenca del río Magdalena, sin fecha. IUQ 349, (113); Antioquia, Anorí; La Rosa, río Cauca; 17 oct 1988. IUQ 350, (77), La Guajira; río Ranchería; 24 ago 1981. IUQ 10, (cuatro), Quindío, río La Vieja, quebrada afluente del río Quindío, 9 ago 1994. IUQ 227, (dos), Valle, cuenca del río La Paila, 15 sep 1996. IUQ 254, (nueve), Quindío, río La Vieja en la balastrera, hacienda Playa Azul, jun 1996. IUQ 272, (uno), Valle del Cauca, Alto Cauca, quebrada San Pablo, afluente río La Paila, 14 sep 1996, IUQ 613, (cuatro), Quindío, río La Vieja, vereda Maravelez, la Tebaida, 25 mar 1991. IUQ 1269, (tres), Quindío, Río La Vieja, quebrada El Macho, afluente del río Barragán, 8 jul 
CUADRO 1

Datos morfométricos y merísticos de Bryconamericus arilepis sp. nov.

Promedio entre paréntesis, longitud estándar y total en $\mathrm{mm}$.

TABLE 1

Morphometric and meristic data of Bryconamericus arilepis sp. nov. Average in parenthesis, Standard and total length in $\mathrm{mm}$.

\begin{tabular}{|c|c|c|}
\hline Morfometría & Holotipo & Paratipos \\
\hline Longitud estándar & 68.01 & $44.72-70.89(58.35)$ \\
\hline Longitud total & 85.28 & $55.51-87.01(71.76)$ \\
\hline \multicolumn{3}{|l|}{ Porcentaje de la longitud estándar: } \\
\hline 1. Profundidad del cuerpo & 29.89 & $24.42-28.60(26.57)$ \\
\hline 2. Longitud hocico-aleta dorsal & 49.73 & $49.73-53.43(50.93)$ \\
\hline 3. Longitud hocico-aleta pectoral & 24.19 & $24.2-26.36(25.0)$ \\
\hline 4. Longitud hocico-aletas pélvicas & 46.13 & $42.41-47.31(45.29)$ \\
\hline 5. Longitud aleta dorsal-aleta pectoral & 38.23 & $38.2-39.00(37.29)$ \\
\hline 6. Longitud hocico-aleta anal & 59.81 & $52.85-61.38(58.38)$ \\
\hline 7. Longitud aleta dorsal-hipurales & 55.46 & $50.0-56.63(52.84)$ \\
\hline 8. Longitud aleta dorsal-aleta anal & 30.35 & $25.72-30.38(28.23)$ \\
\hline 9. Longitud aleta dorsal & 21.44 & $19.32-23.17(21.15)$ \\
\hline 10. Longitud aletas pectorales & 20.73 & $19.37-22.19(20.83)$ \\
\hline 11. Longitud aletas pélvicas & 14.09 & $12.74-20.07(14.21)$ \\
\hline 12.Longitud aleta anal & 15.47 & $14.14-16.9(15.68)$ \\
\hline 13. Profundidad del pedúnculo caudal & 11.33 & $9.32-11.34(10.29)$ \\
\hline 14. Longitud pedúnculo caudal & 9.41 & $9.33-13.67(11.37)$ \\
\hline 15. Longitud cabeza & 21.26 & $21.39-24.17(22.61)$ \\
\hline \multicolumn{3}{|l|}{ Porcentaje de la longitud cabeza: } \\
\hline 16. Longitud hocico & 29.46 & $24.10-33.15(27.63)$ \\
\hline 17. Diámetro del ojo & 32.02 & $31.32-40.76(36.38)$ \\
\hline 18. Longitud postorbital de la cabeza & 40.39 & $33.61-42.14(37.35)$ \\
\hline 19. Longitud del hueso maxilar & 33.4 & $27.88-36.58(32.71)$ \\
\hline 20. Ancho interorbital & 33.06 & $30.16-34.56(32.48)$ \\
\hline 21. Longitud mandíbula superior & 32.09 & $26.46-34.27(31.95)$ \\
\hline \multicolumn{3}{|l|}{ Merística: } \\
\hline Número de escamas en la línea lateral & 52 & $51-54$ \\
\hline Número de escamas entre la línea lateral y la aleta dorsal & 10 & $9-11$ \\
\hline Número de escamas entre la línea lateral y la aleta anal & 9 & $9-11$ \\
\hline Número de escamas entre la línea lateral y las aletas pélvicas & 8 & $9-10$ \\
\hline Número de escamas predorsales & 17 & $16-18$ \\
\hline Número de radios en la aleta dorsal & iii, 7 & iii, $7-8$ \\
\hline Número de radios en la aleta anal & iv, 23 & iii-iv,20,24 \\
\hline Número de radios en las aletas pélvicas & ii,6 & ii,6 \\
\hline Número de radios en las aletas pectorales & ii, 11 & ii, $10-12$ \\
\hline
\end{tabular}


CUADRO 2

Conteos de escamas en Bryconamericus arilepis sp. nov. y las demás especies de Bryconamericus, A: Número de escamas en la línea lateral, B: Número de escamas entre la línea lateral y el origen de la aleta dorsal, C: Número de escamas entre la línea lateral y el origen de la aleta anal; D: Número de escamas entre la línea lateral y el origen de la aletas pélvicas, E: Sumatoria del número escamas entre la aleta dorsal-línea lateral y origen de la aleta anal, F: Sumatoria del número escamas entre la aleta dorsal-línea lateral y origen de las aletas pélvicas; G: Número de escamas predorsales

TABLE 2

Counts of scales in Bryconamericus arilepis sp. nov. and all species of Bryconamericus, A: Lateral line scale number; B: Lateral line scale-dorsal fin origin number, C: Lateral line scale-anal fin origin number; D: Lateral line scale-pelvic fin origin number, E: Scale total number dorsal fin-lateral line and anal fin origin, F: Scale total number dorsal fin-lateral line and pelvic fin origin; $G$ : predorsal scale numbers

\begin{tabular}{|c|c|c|c|c|c|c|c|c|}
\hline No. & Especie & A & B & $\mathrm{C}$ & $\mathrm{D}$ & $\mathrm{E}$ & $\mathrm{F}$ & G \\
\hline 1 & B. alburnus & 37 & 5 & - & - & - & 9 & - \\
\hline 2 & B. alpha & $32-38$ & $5-6$ & $5-6$ & 4 & $10-13$ & $9-11$ & $11-13$ \\
\hline 3 & B. andresoi & $39-41$ & $5-6$ & $5-6$ & 5 & $10-13$ & $10-12$ & $13-15$ \\
\hline 4 & B. arilepis sp. nov. & $50-54$ & $9-11$ & $9-10$ & $9-10$ & $17-20$ & $17-20$ & $16-18$ \\
\hline 5 & B. bayano & $36-40$ & $6-7$ & $5-6$ & $5-6$ & $11-14$ & $11-14$ & $12-14$ \\
\hline 6 & B. bolivianus & $35-38$ & 4 & - & $2-3$ & - & $6-8$ & - \\
\hline 7 & B. brevirostris & 42 & 8 & 8 & 7 & 16 & 15 & - \\
\hline 8 & B. carlosi & $33-36$ & $5-6$ & 4 & $3-5$ & $9-11$ & $8-12$ & $10-11$ \\
\hline 9 & B. caucanus & $35-41$ & $5-7$ & $4-7$ & $4-6$ & $9-15$ & $9-14$ & $9-15$ \\
\hline 10 & B. charalae & $31-34$ & $4-5$ & $3-4$ & 3 & $7-10$ & $7-9$ & $10-12$ \\
\hline 11 & B. cinarucoense & $35-37$ & $4-6$ & $3-4$ & $3-4$ & $7-11$ & $7-11$ & $10-11$ \\
\hline 12 & B. cismontanus & $32-38$ & $4-5$ & $3-5$ & $3-4$ & $7-11$ & $7-10$ & $10-12$ \\
\hline 13 & B. cristiani & $39-42$ & $6-8$ & $5-7$ & $5-7$ & $11-16$ & $11-16$ & $11-13$ \\
\hline 14 & B. dahli & $36-40$ & $6-7$ & $4-7$ & $5-6$ & $10-15$ & $11-14$ & $9-12$ \\
\hline 15 & B. delta & $7-12$ & $6-7$ & $4-5$ & $3-4$ & $10-13$ & $9-12$ & - \\
\hline 16 & B. diaphanus & 37 & $4-5$ & - & 3 & - & $7-10$ & $9-10$ \\
\hline 17 & B. ecai & $36-38$ & $5-6$ & 4 & - & $9-11$ & - & $12-14$ \\
\hline 18 & B. eigenmanni & $38-39$ & $5-6$ & - & $3-4$ & - & $8-11$ & 11 \\
\hline 19 & B. emperador & $37-43$ & $6-8$ & $5-8$ & $6-8$ & $11-17$ & $12-17$ & $11-13$ \\
\hline 20 & B. exodon & $34-43$ & $4-6$ & $3-5$ & - & $7-12$ & - & $11-14$ \\
\hline 21 & B. galvisi & $40-42$ & $5-7$ & $5-7$ & $5-6$ & $10-15$ & $10-14$ & $12-17$ \\
\hline 22 & B. geryi & $39-41$ & $6-7$ & - & 4 & - & $10-12$ & $11-13$ \\
\hline 23 & B. gonzalezoi & $37-39$ & $6-7$ & $5-7$ & $5-6$ & $11-15$ & $11-14$ & $12-13$ \\
\hline 24 & B. grosvenori & $40-43$ & 5 & - & 4 & - & 10 & 15 \\
\hline 25 & B. guaytarae & $38-42$ & $5-7$ & $4-6$ & $4-6$ & $9-14$ & $9-14$ & $11-14$ \\
\hline 26 & B. guizae & $36-40$ & 6 & $5-5$ & $4-5$ & $11-12$ & $10-12$ & $12-13$ \\
\hline 27 & B. heteresthes & $34-36$ & 5 & $3-4$ & 3 & $8-10$ & 9 & $9-10$ \\
\hline 28 & B. huilae & $40-45$ & $6-8$ & $5-7$ & $5-7$ & $11-16$ & $11-16$ & $14-19$ \\
\hline 29 & B. hyphesson & $33-36$ & 4 & - & 3 & - & 8 & - \\
\hline
\end{tabular}


CUADRO 2 (Continuación)

Conteos de escamas en Bryconamericus arilepis sp. nov. y las demás especies de Bryconamericus, A: Número de escamas en la línea lateral, B: Número de escamas entre la línea lateral y el origen de la aleta dorsal, C: Número de escamas entre la línea lateral y el origen de la aleta anal; D: Número de escamas entre la línea lateral y el origen de la aletas pélvicas, E: Sumatoria del número escamas entre la aleta dorsal-línea lateral y origen de la aleta anal, F: Sumatoria del número escamas entre la aleta dorsal-línea lateral y origen de las aletas pélvicas; G: Número de escamas predorsales

TABLE 2 (Continued)

Counts of scales in Bryconamericus arilepis sp. nov. and all species of Bryconamericus, A: Lateral line scale number; B: Lateral line scale-dorsal fin origin number, C: Lateral line scale-anal fin origin number; D: Lateral line scale-pelvic fin origin number, E: Scale total number dorsal fin-lateral line and anal fin origin, F: Scale total number dorsal fin-lateral line and pelvic fin origin; $G$ : predorsal scale numbers

\begin{tabular}{|c|c|c|c|c|c|c|c|c|}
\hline No. & Especie & A & B & $\mathrm{C}$ & $\mathrm{D}$ & E & $\mathrm{F}$ & G \\
\hline 30 & B. hypopterus & $35-38$ & $5-6$ & $4-5$ & $3-4$ & $9-12$ & $8-11$ & $11-13$ \\
\hline 31 & B. ichoensis & $33-36$ & $5-6$ & $4-6$ & $4-5$ & $9-13$ & $9-12$ & $10-11$ \\
\hline 32 & B. iheringii & $37-38$ & $5-6$ & $3-4$ & - & $8-11$ & - & 12 \\
\hline 33 & B. ikaa & $36-38$ & 5 & - & $3-5$ & - & $8-11$ & $12-14$ \\
\hline 34 & B. lambari & $38-40$ & $5-6$ & - & $4-5$ & - & $9-12$ & $11-14$ \\
\hline 35 & B. lassorum & $35-36$ & $5-6$ & $5-6$ & $4-5$ & $10-13$ & $9-12$ & - \\
\hline 36 & B. loisae & $38-41$ & $5-7$ & $5-6$ & $5-6$ & $10-14$ & $10-14$ & $11-14$ \\
\hline 37 & B. macrophtalmus & $40-41$ & 5 & 4 & 3 & 10 & 9 & $10-12$ \\
\hline 38 & B. hemigrammus & $5-10$ & 4 & - & 3 & - & 8 & - \\
\hline 39 & B. meridae & $33-37$ & $4-5$ & $2-3$ & $2-3$ & $6-9$ & $6-9$ & $11-13$ \\
\hline 40 & B. microcephalus & $38-40$ & 5 & - & 4 & - & 10 & $12-13$ \\
\hline 41 & B. miraensis & $39-41$ & $5-6$ & $4-5$ & $4-5$ & $9-12$ & $9-12$ & $14-15$ \\
\hline 42 & B. multiradiatus & $34-37$ & $5-6$ & $4-6$ & $3-6$ & $9-13$ & $8-13$ & $10-13$ \\
\hline 43 & B. novae & 36 & 4 & - & 3 & - & 8 & $9-10$ \\
\hline 44 & B. orinocoense & $31-32$ & 5 & $3-4$ & $3-4$ & $8-10$ & $8-10$ & $9-10$ \\
\hline 45 & B. orteguasae & $35-39$ & $4-5$ & $3-4$ & 3 & $7-10$ & $7-9$ & 11 \\
\hline 46 & B. pachacuti & $33-37$ & 6 & $4-5$ & $3-4$ & $10-12$ & $9-13$ & - \\
\hline 47 & B. patriciae & $37-40$ & $5-6$ & $4-5$ & - & $9-12$ & - & $13-15$ \\
\hline 48 & B. pectinatus & $32-36$ & $6-7$ & $5-6$ & - & $11-14$ & - & - \\
\hline 49 & B. peruanus & $38-40$ & $6-7$ & $5-7$ & $5-7$ & $11-15$ & $11-15$ & $11-14$ \\
\hline 50 & B. phoenicopterus & $37-41$ & $7-8$ & $6-8$ & $5-6$ & $13-17$ & $12-15$ & - \\
\hline 51 & B. plutarcoi & $40-50$ & $6-8$ & $5-6$ & $5-7$ & $11-15$ & $11-16$ & $11-16$ \\
\hline 52 & B. scleroparius & $37-39$ & $6-7$ & $6-7$ & $6-7$ & $12-15$ & $12-15$ & $10-13$ \\
\hline 53 & B. shinahota & $36-38$ & 6 & - & 5 & - & 12 & $11-13$ \\
\hline 54 & B. simus & 39 & 6 & - & 4 & - & 11 & $10-11$ \\
\hline 55 & B. singularis & $30-32$ & $5-6$ & 4 & 4 & $9-11$ & $9-11$ & 10 \\
\hline 56 & B. stramineus & 38 & 5 & - & $3-4$ & - & $8-10$ & - \\
\hline 57 & B. subtilisform & $38-39$ & 4 & 3 & $2-3$ & 8 & $6-8$ & $10-11$ \\
\hline 58 & B. tenuis & $35-37$ & $5-6$ & - & 3 & - & $8-10$ & $12-15$ \\
\hline
\end{tabular}


CUADRO 2 (Continuación)

Conteos de escamas en Bryconamericus arilepis sp. nov. y las demás especies de Bryconamericus, A: Número de escamas en la línea lateral, B: Número de escamas entre la línea lateral y el origen de la aleta dorsal, C: Número de escamas entre la línea lateral y el origen de la aleta anal; D: Número de escamas entre la línea lateral y el origen de la aletas pélvicas, E: Sumatoria del número escamas entre la aleta dorsal-línea lateral y origen de la aleta anal, F: Sumatoria del número escamas entre la aleta dorsal-línea lateral y origen de las aletas pélvicas; G: Número de escamas predorsales

TABLE 2 (Continued)

Counts of scales in Bryconamericus arilepis sp. nov. and all species of Bryconamericus, A: Lateral line scale number; B: Lateral line scale-dorsal fin origin number, C: Lateral line scale-anal fin origin number; D: Lateral line scale-pelvic fin origin number, E: Scale total number dorsal fin-lateral line and anal fin origin, F: Scale total number dorsal fin-lateral line and pelvic fin origin; $G$ : predorsal scale numbers

$\begin{array}{llccccccc}\text { No. } & \text { Especie } & \text { A } & \text { B } & \text { C } & \text { D } & \text { E } & \text { F } & \text { G } \\ 59 & \text { B. terrabensis } & 47-54 & 8-9 & 6-9 & 7-8 & 14-19 & 15-18 & 11-15 \\ 60 & \text { B. thomasi } & 35-37 & 6-7 & 5 & - & 11-13 & - & - \\ 61 & \text { B. tiquiensis } & 38-42 & 5 & - & 3-5 & - & 8-11 & 10-12 \\ 62 & \text { B. tolimae } & 40-45 & 6-8 & 5-8 & 5-7 & 11-17 & 11-16 & 13-17 \\ 63 & \text { B. turiuba } & 37-43 & 4-5 & 7-10 & - & 3-4 & - & 10-15 \\ 64 & \text { B. yokiae } & 39-42 & 7-8 & 6-8 & 5-7 & 13-17 & 12-16 & 12-14 \\ 65 & \text { B. ytu } & 37-39 & 4-6 & - & 4 & - & 8-11 & 12-14 \\ 66 & \text { Bryconamericus sp. } & 36-44 & 5-8 & 5-8 & 5-7 & 10-17 & 10-16 & 11-16\end{array}$

1996. IUQ 1301, (seis), Caldas, río Chamberri, afluente río Cauca, en la India, Salamina ( $5^{\circ} 24^{\prime}$ $55^{\prime \prime} \mathrm{N}$ y $\left.75^{\circ} 30^{\prime} 10^{\prime \prime} \mathrm{W}\right), 2$ jul 2004. IUQ 856, (35), Antioquia, quebrada Los Dragos, afluente del río arriba vereda Francisca-Sacantin, Sonsón. IUQ 870, (32), Antioquia, río Anorí, afluente río Nechi, transecto río vía Anorí-La Punta Anorí ( $7^{\circ} 4$ ' $17^{\prime \prime} \mathrm{N}$ y $75^{\circ} 07^{\prime} 48^{\prime \prime} \mathrm{W}$ a $1499 \mathrm{~m}$ ), 10 sep 2003. IUQ 865, (ocho), Antioquia, quebrada La Chinca, afluente del río Anorí $200 \mathrm{~m}$ de La Punta, 10 sep 2003. IUQ 875, (38), Antioquia, quebrada La Tupia, afluente río Nechi, Anorí, 9 sep 2003. IUQ 862, (29), Antioquia, quebrada La Virgen, afluente río Anorí $100 \mathrm{~m}$ antes de su desembocadura, sector del río vía Anorí, La Punta Anorí, (704' 22” N y $75^{\circ} 07^{\prime} 29^{\prime \prime}$ W a $1489 \mathrm{~m}$ ). IUQ 872, (17), Risaralda, quebrada El Tigre, hacienda Guadalupe, vereda el Tigre, Pereira, 19 jul 2005. IUQ 859, (cinco), Antioquia, quebrada Serrana, afluente del río Anorí, $200 \mathrm{~m}$ de la vía Anorí, La Punta-Anorí, 10 sep 2003. IUQ 858, (diez), Antioquia, río Sonsón $100 \mathrm{~m}$ antes de la represa, Sonsón, jul 18 de 2004. IUQ 301b, (uno) (C. y T.), Quindío, alto río Quindío, alto Cauca, 28 ene 2000. IUQ 851, (seis), río Anori, La Rosa, bajo Cauca, sistema río Magdalena, 17 oct 1988. IUQ 861, (dos) (C. y T.), Antioquia, cuenca del Magdalena, quebrada La Virgen afluente Río Anorí, 100 m antes de su desembocadura, sector del río vía Anorí-La Punta

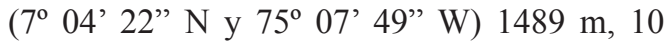
sep 2003. IUQ 863, (dos) (C. y T.), Antioquia, cuenca del Magdalena, río Anorí afluente río Nechí, sector río vía Anorí-La Punta ( $7^{\circ} 4^{\text {' }}$ $17^{\prime \prime} \mathrm{N}$ y $75^{\circ} 07^{\prime}$ 48" O) $1499 \mathrm{~m}, 10$ sep 2003; IUQ 864, (dos) (C. y T.), Antioquia, cuenca del Magdalena, quebrada La Tupía afluente río Nechí, 9 sep 2003. IUQ 868, (dos) (C. y T.), Antioquia, cuenca del Magdalena, quebrada La Chinca afluente río Anorí, 200 m vía Anorí-La Punta, 10 sep 2003. IUQ 879, (uno) (C. y T.), Antioquia, cuenca del Magdalena, Quebrada Serrana afluente río Anorí, $200 \mathrm{~m}$ de la vía Anorí-La Punta, 10 sep 2003. IUQ 943, (uno) (C. y T.), Antioquia, río Sonsón $100 \mathrm{~m}$ antes de 
la represa, Sonsón, cuenca del Magdalena, 18 jul 2004. IUQ 948, uno (C. y T.), Antioquia, quebrada Los Dragos, afluente del río Arriba vereda Francisca-sacantin, Sonsón, cuenca del Magdalena, 18 jul 2004. IUQ 1202, (uno) (C. y T.), Quindío, quebrada Boquia, afluente río Quindío. IUQ 1571, (dos) (C. y T.), Caldas, río Chamberri, afluente río Cauca, en la India, Salamina (5 $24^{\prime} 55^{\prime \prime} \mathrm{N}$ y $75^{\circ} 30^{\prime} 10^{\prime}$ ' W). IUQ 1584, (uno) (C. y T.), La Guajira, río Ranchería, Caribe Colombiano, 20 ago 1981.

Bryconamericus huilae. (IUQ 423 en Román-Valencia, 2003b). IUQ 947, (dos) (C. y T.), Huila, quebrada La Criolla, afluente río Guachino, vereda Guachicos en la vía PitalitoSan Agustín, Alto Magdalena, 13 oct 2001.

Bryconamericus tolimae. (Ver IUQ 484, Román-Valencia, 2004). IUQ 1586, (dos) (C. y T.), Tolima, Ibagué, vereda Pastales, $100 \mathrm{~m}$ antes de Pastales en la vía Ibagué-Juntas, río Combeima, afluente río Magdalena (4³0'19" N y 75 17'46” W) 1586 m, 27 dic 2002.

Bryconamericus plutarcoi. IaVH 3123, (12), Santander, río Suárez; ene 1994. IUQ 1577, (uno) (C \& T), Santander, quebrada Santa Rosa de la desembocadura en el río Suárez $100 \mathrm{~m}$ hacia arriba, sistema del río Magdalena (6 $6^{\circ} 26^{\prime} 09^{\prime \prime} \mathrm{N}$ y $\left.73^{\circ} 18^{\prime} 56^{\prime \prime} \mathrm{W}\right) 825$ m, 24 jun 2001.

Bryconamericus sp. IUQ 919, (63), Santander, Charalà, quebrada La Potrera, afluente río Pienta, cuenca río Fonce, vía Charalà-La laguna, 5 feb 2004. IUQ 921, (44), Santander, Charalá, quebrada El Barro, afluente río Pienta, $200 \mathrm{~m}$ puente vía limites, Encino-Charalà, vía el Encino-Charalà, 6 feb 2004. IUQ 923, (41), Santander, Charalá, quebrada La Sanguina, afluente río Pienta, 5 feb 2004. IUQ 926, (22), Santander, Charalá, río Fonce, afluente del río San Gil, vía San GilCharalà, 31 oct 2003. IUQ 927, (35), Santander, Mogotes, quebrada Guayawata, afluente del río Mogotes en el puente $100 \mathrm{~m}$ antes de su boca, 1 nov 2003. IUQ 937, (tres) (C. y T.), Santander,
Mogotes, quebrada Guayawata afluente río Mogotes en el puente $100 \mathrm{~m}$ arriba de su boca (6 29' 07' N y 72॰ 58' 59' W) $1658 \mathrm{~m}$, en Mogotes, 1 nov 2003. IUQ 944, (dos) (C \& T), Santander, San Gil, río Fonce afluente río San Gil, vía San Gil-Charalá, 31 oct 2003. IUQ 1569, (dos) (C. y T.), quebrada La Potrera, afluente río Pienta, cuenca río Fonce, vía Charalà-La laguna Charalà, 5 feb 2004. IUQ 1570, (dos) (C. y T.), río Mogotes, en la boca quebrada Guayawata $100 \mathrm{~m}$ abajo del puente 1 Km. antes de Mogotes, 7 feb 2004. IUQ 1572, (dos) (C. y T.), Santander, Charalà, quebrada La Sanguina, afluente río Pienta, Charalà, 5 feb 2004. IUQ 1573, (dos) (C. y T.), Santander, Charalà, quebrada El Barro, afluente río Pienta, $200 \mathrm{~m}$ puente vía limites, Encino- Charalà, vía el Encino -Charalà.

\section{RESULTADOS}

Bryconamericus arilepis sp. nov. (Cuadros 1, 2, Figs. 1-3)

Holotipo. IUQ 1917, Macho, 68,867 mm LE. COLOMBIA, Santander, Charalá, vereda La Pontesuela, Magdalena medio, cuenca del Río Fonce, Quebrada La Pontesuela en la boca de la quebrada Coclina, en la escuela Carrillo, vía Cantera a Encino $\left(6^{\circ} 10^{\prime} 42^{\prime \prime} \mathrm{N}\right.$ y los 730 09'44”W) 1457 msnm, 6. feb. 2004, C. Román-V. y R. I. Ruiz-C.

Paratipos. IUQ 920, (tres) colectados con el Holotipo; IUQ 1567, (uno) (C. y T.) colectado con el Holotipo. IUQ 1915, (85), COLOMBIA, Santander, Charalá, vereda La Pontesuela, Magdalena medio, cuenca del Río Fonce, Quebrada La Pontesuela en la boca de la quebrada Coclina, en la escuela Carrillo, vía Cantera a Encino $\left(6^{\circ} 10^{\prime} 42^{\prime \prime} \mathrm{N}\right.$ y los 7309'44"W) 1457 msnm, 17 ene 2008, C. Román-V. y C. A. García.

Diagnosis. Bryconamericus arilepis se distingue de sus congéneres por el mayor número de escamas: sobre la línea lateral (5054 vs. 30-50, excepto B. terrabensis con 47-54 escamas), entre la línea lateral y el origen de la aleta dorsal (9-11 vs. 4-9), entre la línea lateral 


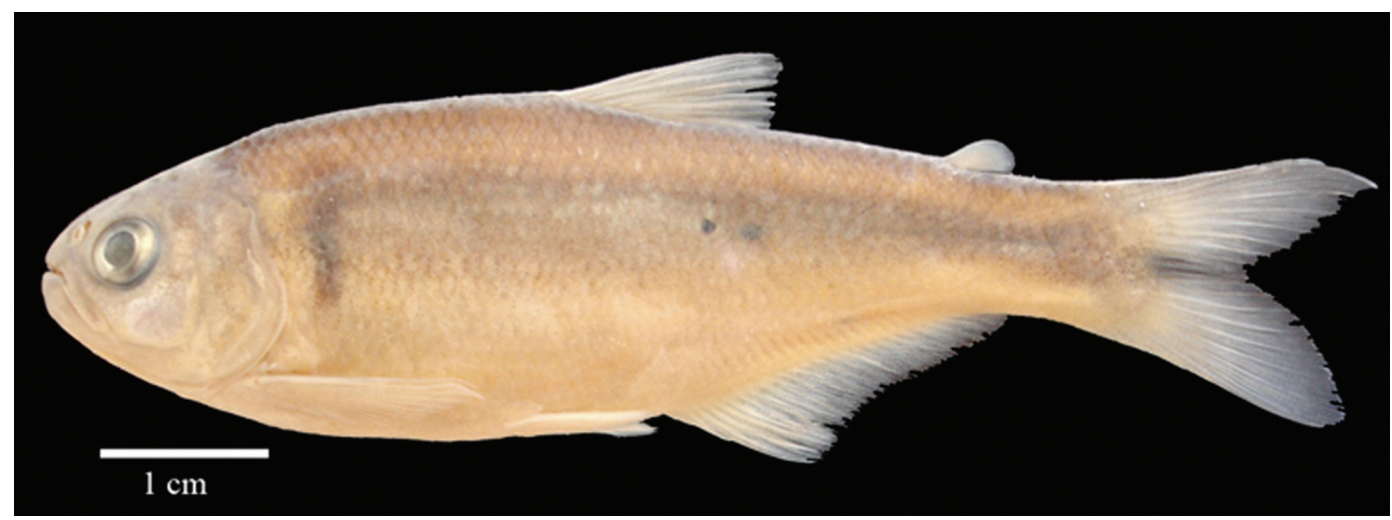

Fig. 1. Bryconamericus arilepis sp. nov. (Holotipo), male, $68.867 \mathrm{~mm}$ LE. Colombia, Santander, Charalá, vereda La Pontesuela, Magdalena medio.

Fig.1. Bryconamericus arilepis sp. nov. (Holotype), male, $68.867 \mathrm{~mm}$ SL. Colombia, Santander, Charalá, locality La Pontesuela, Magdalena median.

y el origen de las aletas pélvicas (8-10 vs. 2-8), entre la línea lateral y el origen de la aleta anal (8-10 vs. 2-8 excepto B. terrabensis con 6-9) y predorsales (16-18 vs. 9-16, excepto $B$. huilae con 14-19 escamas, B. galvisi con 12 a 17 у $B$. tolimae con 13-17).

English diagnosis. Bryconamericus arilepis can be distinguished from all congeners by the higher number of scales: on lateral line (5054 vs. 30-50, except B. terrabensis with 47-54), between lateral line to dorsal fins origin (9-11 vs. 4-9), between lateral line and pelvic fin origin (8-10 vs. $2-8)$, between lateral line and anal fin origin (8-10 vs. 2-8, except $B$. terrabensis with 6-9) and predorsals (16-18 vs. 9-16, except $B$. huilae with 14-19 scales, B. galvisi with 12-17 and B. tolimae with 13-17).

Descripción. Cuerpo corto y robusto en la parte anterior; parte dorsal de las órbitas convexo. Perfil dorsal de la cabeza y del cuerpo curvo desde el hocico hasta el origen de la aleta dorsal, oblicuo desde el último radio de la aleta dorsal hasta la base de la aleta caudal. Perfil ventral del cuerpo convexo desde el hocico hasta la base de la aleta anal, más pronunciada en la parte posterior a las aletas pectorales.
Borde de la aleta dorsal oblicuo, segundo radio simple y los primeros dos radios ramificados más largos.

Pedúnculo caudal lateralmente comprimido en todos los ejemplares. Cabeza y hocico cortos; mandíbulas iguales; boca terminal; labios blandos y flexibles, no cubren externamente la hilera externa de dientes del premaxilar; parte ventral de la mandíbula superior plana; extremo posterior del maxilar sobrepasa el borde anterior de la orbita; abertura de las narinas posteriores verticalmente ovoide; abertura de las narinas anteriores con excrescencia membranosa.

La superficie anterior del frontal separado por el extremo posterior del mesetmoides; extremo anterolateral del frontal no cubre el extremo dorsal cartilaginoso del etmoides lateral. Fontanela frontoparietal ensanchada a nivel del parietal. Rinoesfenoides cartilaginoso, la parte anterior se extiende sobre el borde posterior del prevómer, posteriormente sobre la superficie antero dorsal del paraesfenoides, dorsalmente se proyecta en un proceso hacia el orbitoesfenoides, lateralmente a nivel del etmoides lateral. Orbitoesfenoides unido anteriormente al rinoesfenoides por un proceso laminar a nivel medial, cubierto en el borde 
posterior por una banda de cartílago. Espina supraoccipital corta.

Seis infraorbitales presentes, el primer infraorbital se extiende en la parte anterior sobre el borde dorsal del proceso del maxilar, mientras el borde dorso-posterior se proyecta, en una estructura tubular sobre la parte anterior del segundo infraorbital, el tercer infraorbital con el borde posteroventral en contacto con el preoperculo, cubre el cuadrado, el metapterigoide y el extremo ventral del hiomandibular, el cuarto y quinto infraorbital de igual tamaño y cubren la parte dorsal del hiomandibular, el sexto infraorbital cubre la espina del esfenótico y se proyecta en forma de canal, hasta alcanzar el canal del extremo latero posterior del frontal. Supraorbital ausente. Premaxilar con dos hileras de dientes; hilera externa de cinco dientes con cuatro a cinco cúspides y orientados en línea recta. Hilera interna con cuatro dientes con dos o tres cúspides, con la cúspide central mucho mayor. Maxilar largo, alcanza el borde dorsal del cartílago de Meckel en el dentario y sobrepasa la mitad del segundo infraorbital. Maxilar con 4-8 dientes: entre uni o multicúspides. Dentario con tres dientes frontales grandes pentacúspides, con las cúspides laterales muy pequeñas cuarto diente mediano, pentacúspide, seguido por nueve a diez dientes laterales que disminuyen de tamaño gradualmente y que alternan el número de sus cúspides de adelante para atrás bicúspide, tricúspide y unicúspide.

Ocho supraneurales distribuidos entre la 5 y $12^{\text {va }}$ espina neural, del 2 al 7 delgados, alargados y de estructura ósea con el extremo dorsal cartilaginoso; los supraneurales 1 y 8 son reducidos, el supraneural 1 de estructura cartilaginosa y el 8 óseo. Los pteriogióforos proximales de la aleta dorsal insertados entre las espinas neural 14-21. Con 23 pteriogióforos proximales en la aleta anal. La cintura pectoral con el postemporal largo y delgado, extendido sobre el borde dorso posterior del opérculo, su extremo ventral cubre el proceso dorsal del supracleitrum, y está en contacto con el primer postcleitrum. Cuatro radiales proximales. Aleta pélvica corta, sus extremos no alcanzan el origen de la aleta anal. Hueso pélvico alargado, borde lateral recto con punta anterior de cartílago, extremo posterolateral cartilaginoso, superficie interna cóncava, elemento radial cartilaginoso, proceso isquiático óseo, corto, curvo, prolongado en un proceso puntiagudo de cartílago.

Aleta caudal bifurcada con lóbulos cortos y puntiagudos. Radios caudales principales 10/8. Radios caudales procurrentes $9 / 11$. Aleta caudal sin escamas, excepto en su base. Con 50-54 escamas con poros de la línea lateral. Poros de la línea lateral forma una leve curva entre la primera y la octava-novena escama, el resto en línea recta. Número total de vértebras 42-43.

Dimorfismo sexual secundario. Los machos tienen una hilera de espinas cortas sobre el primero al quinto o sexto radio ramificado de la aleta anal, su distribución se da con nueve a 14 espinas, ubicadas sobre la parte izquierda y anterior de la ramificación. Sobre el segundo al quinto radio ramificado y sobre el último radio simple de la aleta pélvica, con nueve a 14 espinas, ubicadas sobre cada segmento en las ramificaciones de la derecha, no alcanzan el extremo anterior. No se observaron espinas en los radios simples.

Color en vivo. Área dorsal verde-oscuro, lateral blanco plateado, más pronunciado en la parte ventral; parte lateral del cuerpo con tonalidad verde- amarillento; mancha humeral oscura verticalmente alargada; mancha opercular morada; una banda lateral oscura se prolonga sobre los radios medios caudales; aletas pectorales con radios oscuros; aletas pélvicas hialinas; base de la aleta dorsal amarilla, con una banda oscura en la parte distal; aleta anal oscura en extremo distal de radios, hialinos en su base; lóbulos de la aleta caudal amarilloclaro en su base y parte media; hialinos en sus extremos.

Distribución. Conocida sólo en su localidad típica en la quebrada Pontesuela. 
Aspectos ecológicos. Datos obtenidos en enero 6 de 2004 a las 8:10 hr. Temperatura del aire $15.9^{\circ} \mathrm{C}$, temperatura del agua $17.8^{\circ} \mathrm{C}$, oxígeno disuelto $6.4 \mathrm{mg} / \mathrm{l}$, saturación $80 \%$, pH 7.6. Ancho 1-2 m, profundidad 0.30-0.50 $\mathrm{m}$, sustrato conformado por detrito, piedra y material vegetal en descomposición, agua oscura. Mientras en enero 17 de 2008 a las 9:11 hr. los datos son los siguientes: temperatura del agua $19.8^{\circ} \mathrm{C}$, no hay dato de oxígeno, $\mathrm{pH}$ 6.8, conductividad 54 us/ $\mathrm{cm}$, sólidos totales disueltos $27 \mathrm{ppm}$., ancho 1.5-3.0 m, profundidad 0.86-1.0 m, agua café-oscuro. La dieta observada, en dos estómagos arrojó lo siguiente: material vegetal $(1.75 \%)$, restos de insectos (7.61\%), Odonata (1.69\%); algas: Oedogoniales (2.33\%), Tetrasporaceae $(70.76 \%)$, Desmidiaceae (6.43\%), Bacillariophyceae $(2.33 \%)$ y Oscillariophyceae (7.01\%). El tamaño osciló entre 11.6-16.10 mm, largo 5.54-8.51 mm, se observaron de cinco a seis ciegos pilóricos.
Etimología. El nombre del nuevo taxon deriva de la raíz griega ari $=$ muchas y lepis $=$ escama, alusiva al alto número en sus escamas.

Comentarios. El gráfico de cajas permitió identificar un valor atípico para $B$. caucanus en el número de escamas con poros en la línea lateral (44) (Fig. 2). Se contrasta con Bryconamericus arilepis, el cual se distinguió por su elevado número de escamas (50-54), muy por encima de los valores en las demás especies conocidas (Cuadro 2). Lo cual se constata mediante el gráfico de medias con un intervalo de confianza del 99\% (Fig. 3). En general, cada una de las especies y poblaciones de la cuenca del río Magdalena tiene una probabilidad del $99 \%$ de ser caracterizada por su número de escamas en la línea lateral (Fig. 3). B. arilepis comparte las sinapomorfías propuestas por Vanegas-Ríos (2008) para soportar la monofilia de las especies de Bryconamericus de Centro América y noroccidente de Suramérica.

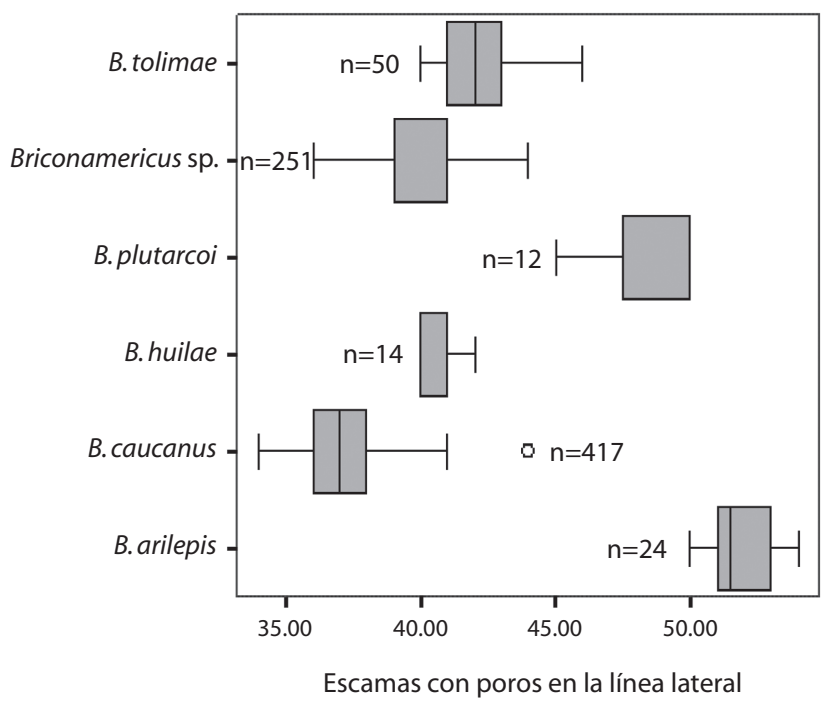

Fig. 2. Variación de las escamas con poros en la línea lateral de B. arilepis sp. nov. y especies de la cuenca del Magdalena.

Fig. 2. Scale pored on lateral line variation of $B$. arilepis sp. nov. and species from Magdalena basin. 


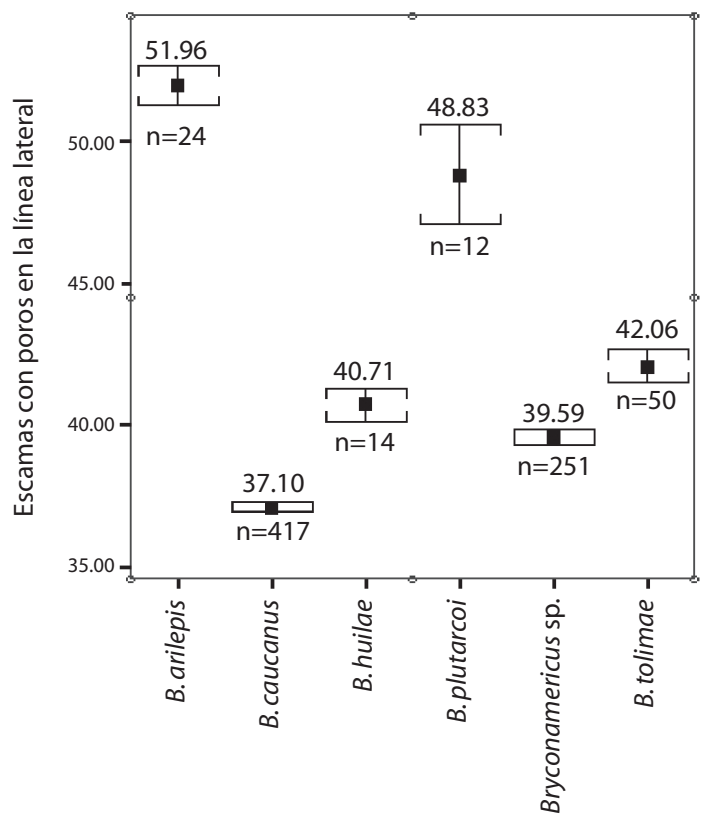

Fig. 3. Valores medios y probabilidad al 99\% de confianza (líneas horizontales) del número de escamas con poros en la línea lateral para B. arilepis sp. nov. y afines de la cuenca del río Magdalena.

Fig.3. Mean data and probability to $99 \%$ (horizontal line) of lateral line scale number of $B$. arilepis sp. nov. and species from Magdalena basin.

\section{Clave taxonómica de las especies de Bryconamericus de Colombia}

\section{Clave a las especies de Bryconamericus de la vertiente pacífico}

1. Seis a siete dientes en la primera hilera del premaxilar . . . . . . . . . . . B. dahli

1.1. Tres a seis dientes en la primera hilera del premaxilar $\ldots \ldots \ldots \ldots \ldots \ldots \ldots \ldots \ldots$

2. En línea recta la hilera interna de dientes del premaxilar $\ldots \ldots \ldots \ldots \ldots \ldots \ldots \ldots$

2.2. No en línea recta la hilera interna de dientes del premaxilar . . . . . . . . . . . . . .5

3. Tres grandes dientes y ocho a nueve pequeños dientes en el dentario; mancha peduncular

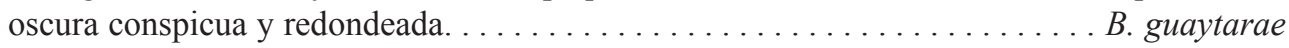

3.3. Más de tres grandes dientes y menos de ocho o nueve pequeños dientes en el dentario; mancha peduncular no oscura conspicua y redondeada $\ldots \ldots \ldots \ldots \ldots \ldots \ldots \ldots \ldots$

4. Extremos de las aletas pectorales no sobrepasan el origen de las aletas pélvicas; borde posterior del tercer infraorbital oblicuo; cuatro dientes en la hilera externa del premaxilar ....

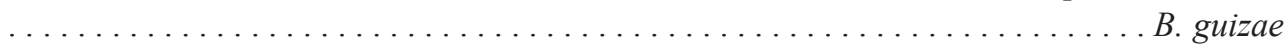

4.4. Extremos de las aletas pectorales sobrepasan el origen de las aletas pélvicas; borde posterior del tercer infraorbital recto; cinco dientes en la hilera externa del premaxilar . . B. andresoi

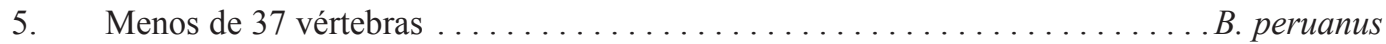

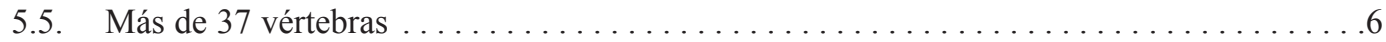

6. 10 a 13 escamas predorsales; 6-8 escamas entre el origen de las aletas pélvicas y la línea

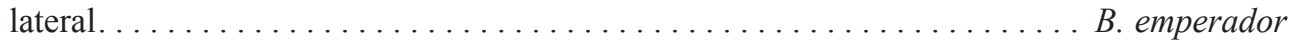


6.6. 14 a 15 escamas predorsales; 4-5 escamas entre el origen de las aletas pélvicas y la línea

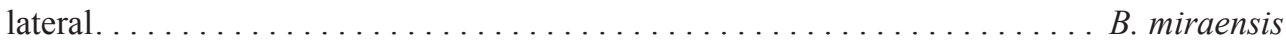

\section{Clave a las especies de Bryconamericus del sistema Río Atrato}

1. Longitud estándar máxima $40 \mathrm{~mm}$; 7-8/7-8 radios procurrentes en la aleta caudal; cero a cuatro dientes pequeños sobre cada dentario $\ldots \ldots \ldots \ldots \ldots \ldots \ldots \ldots \ldots \ldots \ldots \ldots \ldots \ldots \ldots \ldots$

1.1. Longitud estándar máxima mayor de $40 \mathrm{~mm} ; 10 / 10$ radios procurrentes en la aleta caudal; cinco a seis dientes pequeños sobre cada dentario . . . . . . . . . . . . .

2. Cinco a seis radios ramificados en la aleta dorsal; tres grandes dientes sobre cada dentario; profundidad del cuerpo 24.24-27.58\% (media 25.48\%) en el largo estándar; longitud de la cabeza 22.81-25.0\% (media 24.04\%); longitud aletas pectorales $18.71-21.79 \%$ (media $20.71 \%$ ) en el largo estándar. . . . . . . . . . . . . . . . . multiradiatus

2.2. Siete a ocho radios ramificados en la aleta dorsal; ocho a nueve grandes dientes sobre cada dentario; profundidad del cuerpo 26.70-33.58\% (media 29.87\%) en el largo estándar; longitud de la cabeza $25.86-29.76 \%$ (media $26.91 \%$ ); longitud aletas pectorales $24.38-30.22 \%$ (media 26.75\%) en el largo estándar . ....................... ichoensis

\section{Clave a las especies de Bryconamericus del sistema Río Magdalena, Sinú, vertiente Caribe-Guajiro}

1. Menos de 40 escamas en la línea lateral; 37-38 vértebras en total; 10-14 escamas predorsales; machos con espinas grandes en los radios de la aleta anal a partir del primero más largo simple hasta el séptimo u décimo ramificado, se localizan alrededor de la parte media y superior

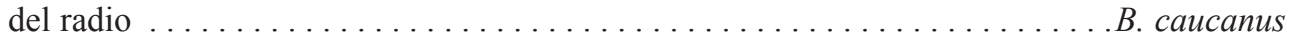

1.1. Más de 40 escamas en la línea lateral; 40-42 vértebras en total; 14-18 escamas predorsales; machos con espinas en la aleta anal distribuidas en casi todos los radios en su parte distal o distinta al anterior . . . . . . . . . . . . . . . . . . . . . . .

2. 50 a 54 escamas con poros en la línea lateral, 9-10 escamas entre el origen de la aleta dorsal y la línea lateral, 8-9 escamas entre la línea lateral-origen de las aletas anal y pélvicas, la suma de las escamas del origen la aleta dorsal a la línea lateral, más la de la línea lateral y el origen de la aleta anal a la línea lateral es $17-21 \ldots \ldots$. . Bryconamericus arilepis sp. nov.

2.2 40-50 escamas con poros en la línea lateral, 6-8 escamas entre el origen la aleta dorsal y la línea lateral, 5-8 escamas entre la línea lateral-origen de las aletas anal y pélvicas, la suma de las escamas de la aleta dorsal a la línea lateral, más la de la línea lateral y la aleta anal a

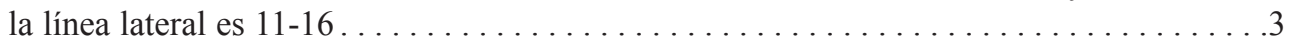

3. Más de diez dientes en cada dentario; mancha humeral con la parte superior e inferior de igual tamaño, de forma rectangular; 23-26 radios ramificados en la aleta anal. . B. plutarcoi

3.3. Menos de diez dientes en cada dentario; mancha humeral en la parte superior e inferior de diferente tamaño, de forma triangular; 18-23 radios ramificados en la aleta anal . . . . . .4

4. 20 pterigióforos proximales en la aleta anal; tres a cuatro dientes en la fila externa del premaxilar; cuatro dientes grandes sobre cada dentario; más de diez radios procurrentes dorsales en la aleta caudal; sin mancha peduncular en ejemplares vivos; machos sin espinas en aletas dorsal y pectorales. . . . . . . . . . . . . . . . . . . . . . . . . .

4.4. 21-23 pterigióforos proximales en la aleta anal; cuatro a cinco dientes en la fila externa del premaxilar; cinco a seis grandes dientes sobre cada dentario; menos de diez radios procurrentes dorsales en la aleta caudal; con mancha peduncular oscura en ejemplares vivos; machos con espinas en aletas dorsal y pectorales B. tolimae 


\section{Clave a las especies de Bryconamericus del sistema Río Orinoco \& Río Catatumbo}

1. 13-18 radios ramificados en la aleta anal; maxilar con dientes serrados; menos de cinco escamas entre la línea lateral y el origen de la aleta dorsal ............ B. cismontanus

1.1. 24-30 radios ramificados en la aleta anal; maxilar sin dientes serrados; más de cinco escamas entre la línea lateral y el origen de la aleta dorsal . . . . . . . . . . . . . . . . 2

2. Dos-tres dientes multicúspides en el maxilar; cuatro o menos dientes pequeños detrás de la fila principal sobre el dentario $\ldots \ldots \ldots \ldots \ldots \ldots \ldots \ldots \ldots \ldots \ldots \ldots \ldots \ldots \ldots \ldots$

2.2. Más de tres dientes en el maxilar con uno a cuatro cúspides; cinco o más pequeños dientes detrás de la fila principal sobre el dentario. . . . . . . . . . . . . . . . . .

3. Orientación en línea recta de los dientes en la hilera externa del premaxilar; seis a ocho dientes pequeños que siguen a los dientes grandes sobre cada dentario . . . . . . B. loisae

3.3. Orientación en zigzag de los dientes en la hilera externa del premaxilar; ocho a 11 dientes pequeños que siguen a los dientes grandes sobre cada dentario . . . . . . . . B. cristiani

\section{Clave a las especies de Bryconamericus del sistema Río Amazonas}

1. Dientes unicúspides a tetracúspides presentes en el maxilar, al menos los primeros y anteriores; 39-40 vértebras en total; 40-42 escamas en la línea lateral; no hay escamas pequeñas sobre radios caudales $\ldots \ldots \ldots \ldots \ldots \ldots \ldots \ldots \ldots \ldots \ldots \ldots \ldots \ldots \ldots \ldots$ galvisi

1.1. Dientes con más de cuatro cúspides en el maxilar; menos de 39 vértebras en total; menos de 40 escamas en la línea lateral, y con escamas pequeñas sobre los radios caudales. . . . . . 2

2. Dientes en la fila externa del premaxilar orientados en línea recta; profundidad del cuerpo mayor a $32.2 \%$ en el largo estándar; cuerpo robusto, aleta dorsal se ubica en la mitad del cuerpo; $32-34$ vértebras en total . . ........................ carlosi

2.2. Dientes en la fila externa del premaxilar no en línea recta; profundidad del cuerpo menos de $32.2 \%$ en el largo estándar; cuerpo alargado; aleta dorsal no se localiza en la mitad del

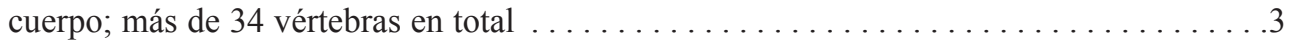

3. Con 14-16 radios ramificados en la aleta anal . . . . . . . . . . . . . orteguasae

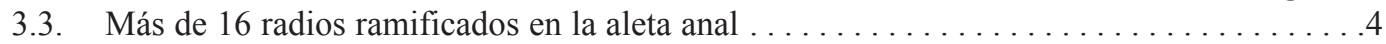

4. Nueve a diez escamas predorsales; 34-36 escamas en la línea lateral; profundidad del cuerpo 23.8-25.0\% en el largo estándar (media 24.3\%); longitud de la aleta dorsal en el largo estándar 18.7-22.7\% (media 20.8\%); con banda lateral plateada y delgada . . . . . B. heteresthes

4.4. 11 o más escamas predorsales; 36-39 escamas en la línea lateral; profundidad del cuerpo 24.5-32.2\% en el largo estándar (media 28.9\%); longitud de la aleta dorsal 21.1-29.1\% en el largo estándar (media 24.23\%); con banda lateral plateada y gruesa . . . . . B. hypopterus

\section{AGRADECIMIENTOS}

Se recibió financiación de la Universidad del Quindío-Vicerrectoria de investigaciones (proyectos 212 y 304) y de la Fundación para la Promoción de la Investigación y la Tecnología del Banco de la República. Idea Wild donó reactivos para laboratorio y equipo de campo. A las siguiente personas por el préstamo de material de comparación: Francisco Provenzano y
Alberto Mercano (MBUCV), José E Castillo y Fabio Quevedo A. (IavH), Mary A. Rogers y Barry Chernoff (FMNH). Donald C. Taphorn (USA) corrigió el resumen en inglés y Carlos A. García (IUQ) elaboró la figura 1.

\section{RESUMEN}

Con base en una serie de 90 ejemplares, en caracteres morfométricos, merísticos y osteología, se describe 
una nueva especie de Bryconamericus de la Quebrada La Pontesuela, boca de la quebrada Coclina, cuenca media del río Magdalena en Colombia. Se utiliza un gráfico de cajas con intervalos de confianza al $99 \%$ para ilustrar la medida merística que mostrara menor solapamiento y distinguiera parcialmente al nuevo taxon de las otras especies del río Magdalena. La especie se distingue de sus congéneres por el mayor número de escamas: sobre la línea lateral (50-54 vs. 30-50, excepto $B$. terrabensis con 47-54 escamas), entre la línea lateral y el origen de la aleta dorsal (9-11 vs. 4-9), entre la línea lateral y el origen de las aletas pélvicas (8-10 vs. 2-8), entre la línea lateral y el origen de la aleta anal (8-10 vs. 2-8 excepto $B$. terrabensis con 6-9) y predorsales (16-18 vs. 9-16, excepto $B$. huilae con 14-19 escamas, $B$. galvisi con 12 a 17 y B. tolimae con 13-17).

Palabras clave: Bryconamericus sp. nov., pez carácido, Magdalena, Sudamérica.

\section{REFERENCIAS}

Almirón, A.E., M.M. Azpelicueta \& J.R. Casciotta. 2004. A new species of Bryconamericus from the arroyo Shangay, río Uruguay basin, Argentina (Teleostei: Characiformes: Characidae). Zool. Abh. 54: 3-10.

Bizerril, C.R.S.F. \& P.M. Auraujo. 1992. Description a une nouvene espece au genre Bryconamericus (Characidae, Tetragonopterinae) du Brésil oriental. Reevue fr. Aquariol. 19:65-69.

Casciotta, J. R., Almiron, A. E. \& Azpelicueta, M. M. 2004. Bryconamericus ikaa, a new species from tributaries of the río Iguazú in Argentina (Characiformes, Characidae). Ichthyol. Explor. Fresh. 15: 61-66.

Eigenmann, C.H. 1927. The American Characidae. Mem. Mus. Comp. Zool. XLIII: 311-358; 417-428.

Ferreira, K.M. \& F.C.T. Lima. 2006. A new species of Knodus (Characiformes: Characidae) from the Rio Tiquié upper Rio Negro system, Brazil. Copeia 4: 630-639.

Ferreira, K. M. \& F. M. Carvajal. 2007. Knodus shinahota (Characiformes: Characidae) a new species from the río Shinahota, río Chapare basin (Mamoré system), Bolivia. Neotrop. Icthyol. 5: 31-36.

Fink, W.L. \& S.H. Weitzman. 1974. The so-called Cheirodontin fishes of Central America with descriptions of two new species (Pisces: Characidae) Smith. Contr. Zool. 172: 1-45.

Langeani, F., Z.M.S. De Lucena, J.P. Lima, \& F.J. TarelhoPereira. 2005. Bryconamericus turiuba, a new species from the upper Rio Paraná system (Ostariophysi: Characiformes). Copeia 2: 386-392.

Lima, F.T.C., L.R. Malabarba, P. A. Buckup, J.F.P. Da Silva, R.P. Vari, A. Harold, R. Benine, O.T. Oyakawa, C.S. Pavaneli, N.A. Menezes, C.A.S Lucena, M.C.S.L. Malabarba, Z. M. S. Lucena, R.E. Reis, F. Langeani, L. Cassati, V.A, Bertaco, C. Moreira \& P.H.F Lucinda. 2003. Genera Incertae Sedis in Characidae, p. 106-169. In: R.E. Reis, S.O. Kullander \& Jr.C.J. Ferraris (eds.). Check list of the freshwater fishes of South and Central America. Edipucrs, Porto Alegre, Brasil.

Lima, F.C.T., H.A. Britski \& F.A. Machado. 2004. New Knodus (Ostariophysi: Characiformes: Characidae) from the upper Rio Paraguay Basin, Brazil. Copeia 3: $577-582$

Malabarba, L.R. \& A. Kindel. 1995. A new species of the genus Bryconamericus Eigennman, 1907 from southern Brazil (Ostariophysi: Characidae). Proc. Biol. Soc. Wash. 108: 679-686.

Miquelarena A.M. \& A.E. Aquino. 1995. Situación taxonómica y geográfica de Bryconamericus thomasi (Teleostei, Characidae). Rev. Brasil. Biol. 55: 559569.

Murray, A.M. 2004. Osteology and morphology of the characiform fish Alestes stuhlmannii Pfeffer, 1896 (Alestidae) from the Rufiji River basin, east Africa. J. Fish Biol. 65: 1412-1430.

Román-Valencia, C. 2002. Revisión sistemática de las especies del género Bryconamericus (Teleostei: Characidae) de Centroamérica. Rev. Biol. Trop. 50: 173-192.

Román-Valencia, C. 2003a. Sistemática de las especies Colombianas de Bryconamericus (Characiformes, Characidae). Dahlia (Rev. Asoc. Colomb. Ictiól) 6:17-58.

Román-Valencia, C. 2003b. Three new species of the genus Bryconamericus (Teleostei: Characidae) from Venezuela. Dahlia (Rev. Asoc. Colomb. Ictiól.) 6: $7-15$.

Román-Valencia, C. 2003c. Description of a new species of Bryconamericus (Teleostei: Characidae) from the Amazon. Boll. Mus. Reg. Sci. nat. Torino, 20: $477-$ 486.

Román-Valencia, C. 2004. Redescripción de Bryconamericus tolimae (Pisces: Characidae), especie endémica del 
río Combeima, cuenca del río Magdalena, Colombia. Dahlia (Rev. Asoc. Colomb. Ictiól) 7: 23-27.

Román-Valencia, C. 2005. Sinopsis comentada de las especies del género Bryconamericus (Teleostei: Characidae) de Venezuela y norte del Ecuador, con la descripción de una nueva especie para Venezuela. Mem. Fund. La Salle de Cienc. Nat., Caracas 163: 27-52.

Román-Valencia, C., D.C. Taphorn \& R.I. Ruiz-C. 2008. Two new Bryconamericus (Characiformes: Characidae) from the Cinaruco River, Orinoco Basin, with keys to all Venezuelan species. Animal Biodiversity and Conservation 31 , in press.

Ruiz-C. R.I. \& C. Román-Valencia. 2006. Osteología de Astyanax aurocaudatus Eigenmann, 1913 (Pisces, Characidae), con notas sobre la validez de Carlastyanax Géry, 1972. Animal Biodiversity and Conservation 29.1: 49-64.
Taylor, W.R. \& G.C. Van Dyke. 1985. Revised procedures for staining and clearing small fishes and other vertebrates for bone and cartilage study. Cybium 9:107-119.

Vanegas-Ríos, J.A. 2008. Filogenia y biogeografía de las especies de peces pertenecientes al género Bryconamericus (Characiformes, Characidae) para América central y noroccidente de América del sur. Trabajo de grado, Facultad de Ciencias Básicas y Tecnologías, Programa de Biología, Universidad del Quindío, Armenia, Colombia.

Vari, R.P. \& Siebert, D.J. 1990. A new, unusually sexually dimorphic species of Bryconamericus (Pisces: Ostariophysi: Characidae) from the peruvian amazon. Proc. Biol. Soc. Wash. 103: 516-524.

Weitzman, S.H. 1962. The osteology of Brycon meeki, a generalized characid fish, with an osteological definition of the family. Stanford Ichthyol. Bull. 8: 1-50. 
